\title{
Balance control near the limits of stability in young soccer players
}

\author{
DOI: https://doi.org/10.5114/pq.2017.74702
}

\section{Piotr Zając ${ }^{1}$, Michał Kuczyński², Ewa Bieć ${ }^{1}$}

${ }^{1}$ University School of Physical Education in Wroclaw, Wroclaw, Poland

${ }^{2}$ Opole University of Technology, Poland

\section{Abstract}

Introduction. To compare postural performance and strategies in young soccer players between quiet standing and standing close to the limits of stability.

Methods. The study included sixteen boys aged 13 years, who have trained soccer for about 5 years. Their body balance was assessed using the Kistler force platform. All participants performed 6 trials, lasting 20 seconds each: three with eyes open and three with eyes closed. The trials included quiet stance, the maximum foreward tilt and maximum backward tilt. Based on the center of pressure (COP) recorded at sampling rate of $100 \mathrm{~Hz}$ the following sway parameters were evaluated: variability, range and mean velocity as well frequency, entropy and fractal dimension.

Results. In frontal and sagittal plane, the COP variability, range and velocity during stances near the limits of stability were higher than in quiet stance. These increases were independent of the direction of body tilt. Body tilts decreased entropy and frequency in the frontal plane and increased fractality in the sagittal plane regardless the tilting direction. There was also a decrease in fractality in the frontal plane that took place during forward tilt only.

Conclusions. Forward and backward tilt deteriorated postural performance in a similar way. However their effects on postural strategies were direction dependent. The strategies in the frontal plane indicated increased investment of attention, lower automaticity and more stable control. In contrast, the sagittal strategies displayed decreased stability with no changes in automaticity. Such inter-plane differences suggest specific allocation of the critical postural tasks in response to the threat of fall. It is plausible that, due to complexity of these reciprocal relationships, testing postural control near to stability limits may better differentiate specific groups and experimental manipulations than testing the limits of stability only.

Key words: postural sway, quiet stance, entropy, fractal dimension, postural strategies

\section{Introduction}

Soccer is the most popular sport in Poland and in the world. People playing that discipline have better balance control compared to some other sports such as basketball or swimming $[1,2]$. In soccer it is particularly important to be able to maintain a vertical posture, often in difficult conditions, for example close to the limits of stability. It has been also shown that players from the higher levels are characterized by better stability, what confirms the importance of an adequate balance in that sport [3, 4]. Better stability contributes to reduced risk of falling and subsequent injury [5]. In addition children who play soccer develop balance control much faster than their untrained peers [6].

Many researchers have already studied athlete's ability to maintain balance. In such experiments, postural performance is usually challenged by occluding vision, standing on unstable surfaces, or reducing the area of the base of support $[7,8]$. Despite such a wide variety of equilibrium assessment conditions, there is still limited data about balance near the limits of stability (LOS). LOS is the amount of maximum excursion an individual is able to intentionally cover in any direction without losing his/her balance or taking a step [9, 10]. It is expected that such conditions may help reveal deficits that are masked under normal conditions due to significant redundancy of the balance control system. Such testing can be very helpful for athletic trainers, physiotherapists and researchers. Balance assessment using this protocol may sig- nificantly contribute to the assessment of postural control in soccer players and other athletes who often approach the limits of stability during game.

Assessment of balance ability is most commonly used to prevent falls, which often result in injuries [5]. When monitoring the recovery, even after the negative consequences of imbalances, we often use this type of measurement. Balance studies are used in many areas, from physiotherapy through sport to occupational medicine [11]. Thanks to the information provided by such tests, serious consequences of uncontrolled falls may be at least partially prevented. For this reason, more and more posturographic research is being undertaken in athletes practicing different sports [12, 13]. Some drawbacks of current studies include too little attention paid to the early stages of sports training as well as overlooking the significance of changes in postural behavior in situations that are hazardeous for stability.

Adolescent athletes have very good postural control which, according to some studies, matches that of young adults $[6,12]$. Therefore the conditions, under which their ability to maintain balance is investigated, should be more complex than those used to evaluate sedentary subjects, older people or individuals with balance control disorders. For the purpose of this preliminary study a convenience sample of adolescent soccer players was selected. Because of their age, sport discipline and specific training, they represent an interesting group for studying postural control during tasks that challenge body stability. The aim of the study was to assess pos-

Correspondence address: Piotr Zając, Katedra Fizjoterapii w Dysfunkcjach Narządu Ruchu, Zakład Diagnostyki Funkcjonalnej, University School of Physical Education in Wrocław, al. I.J. Paderewskiego 35, 51-612 Wrocław, Poland, e-mail: mgrpiotrzajac@gmail.com 
tural performance and strategies in young soccer players at the limits of stability in reference to quiet stance and to examine if these expected changes depend on the availability of vision

\section{Material and methods}

Sixteen 13-year old boys participated. They have been training soccer for 5-6 years at Lechia Dzierżoniów soccer club. The average body weight of the young football players was $48.5 \pm 7.2 \mathrm{~kg}$, while the average body height was $163.5 \pm$ $8.2 \mathrm{~cm}$.

The tests were performed using the Kistler 9286 AA posturographic platform with the sampling rate of $100 \mathrm{~Hz}$. A computer with Bioware software was also used for the analysis of measurements made on the Kistler plate. Based on the recorded forces exerted on the plate, the center of pressure (COP) time-series were calculated.

Prior to the test, each participant was informed about the course of the study, and about the position of the body to be adopted when performing the various parts of the research experiment. All participants performed two blocks of three trials, one with eyes open and one with eyes closed. The trials included quiet stance, the maximum foreward tilt and maximum backward tilt (Figure 1). Each block started with quiet stance, while the sequence of blocks and tilting directions were counterbalanced. The subjects were tested barefoot with the feet parallel and $10 \mathrm{~cm}$ apart and with the arms at the sides. During eyes open test, they were asked to focus their gaze at a marker located 2 meters ahead of them at a height of 1.4 meters. During body tilts, they were instructed to maintain constant contact of their feet with the support surface. They were also instructed to stand as still as possible.

To evaluate postural performance and strategies the following stabilographic parameters were analyzed:

- COP variability [mm] - measured by standard deviation of the COP, characterizes the dispersion of sway around its mean value and is the formal measure of postural performance. Larger COP variability accounts for interior or deteriorated performance usually caused by the task difficulty or insufficient abilities to cope with the task.

- COP range [mm] - is the difference between the largest and the smallest value of the recorded time series. Its meaning is similar to that of COP variability. An added value of this index is its ability to describe the consistency in postural regulation and robustness of the postural control system.

- COP mean velocity [mm/s] - is computed as the COP length devided by the measurement time and is usually interpreted as the activity of postural control system. Higher velocity values are associated with more active and energy demanding control which often occurs in the presence of incompatibility between the task requirements and the performer skill. However, the apparent link between this index and the COP variability and range can be deceptive. Fine postural performance (lower range and variability) can be accomplished at the cost of brisk control (higher velocity), while weak performance (higher range and variability) may be accompanied by insufficient level of sway activity which is often the case in older persons or after fatiguing exercises.

- COP frequency $[\mathrm{Hz}]$ - is computed as a mean frequency and depends on the rate of changing direction by the COP. Increased/decreased frequency significantly contributes to the increase/decrease of mean velocity, provided no changes in sway variability exist.

- COP sample entropy [-] - is a measure of the irregularity
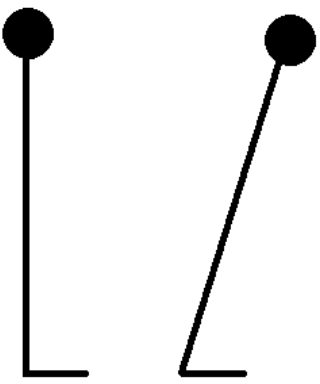

1.

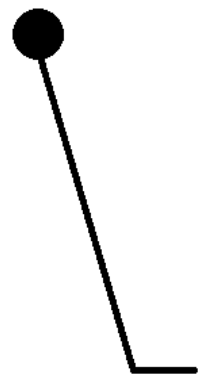

3.
Fig. 1. Three balance task: quiet stance (1.), maximum forward tilt (2.), maximum backward tilt (3.)

attention devoted to controlling balance. Higher entropy values (characteristic for more irregular COP time-series) indicate less attention invested in postural stability, i.e. a more automatic postural control.

- COP fractal dimension [-] - describes the the COP complexity which is most often interpreted in terms of postural stability and adaptability. Specifically, increase in sway fractality is associated with a more adaptive behavior, while decrease indicates the tendency to stabilization.

Selection of the above parameters was justified by their high sensitivity to COP changes, as well as their common acceptance in posturographic research $[14,15]$.

\section{Statistical analysis}

Statistical analysis of the obtained results was performed using Statistica 12. The normality of the distribution of variables was examined by the Shapiro-Wilk test. If the distribution of variables was different than normal, the logarithmic transformation was performed to obtain a normal distribution of variables. An analysis of variance (ANOVA) with repeated measurements was applied, taking into account visual control (eyes open andclosed), position (quiet stance, maximum forward tilt, maximum backward tilt), and plane (frontal and sagittal). The significance of the differences between selected pairs of variables (forward and backward tilt vs. quiet stance) was examined by Tukey's post-hoc test. The level of statistical significance was set at $p<0.05$.

\section{Ethical approval}

The research related to human use has been complied with all the relevant national regulations and institutional policies, has followed the tenets of the Declaration of Helsinki, and has been approved by the authors' institutional review board or an equivalent committee.

\section{Informed consent}

Informed written consent has been obtained from the patients included in this study as well as from their parents.

\section{Results}

\section{COP Variability}

There were three main effects: VISION $(F(1,15)=46.76$, $p<0.001)$, POSITION $(\mathrm{F}(2,30)=20.11, p<0.001)$ and PLANE $(F(1,15)=34.94, p<0.001)$. Variability was larger with eyes closed, at the stability borders, and in the AP plane. Post-hoc test showed that all changes in variablity were significant $(p<0.02)$, except in forward tilt in the AP plane with eyes open. Mean values of COP variability are shown in Figure 2 . 


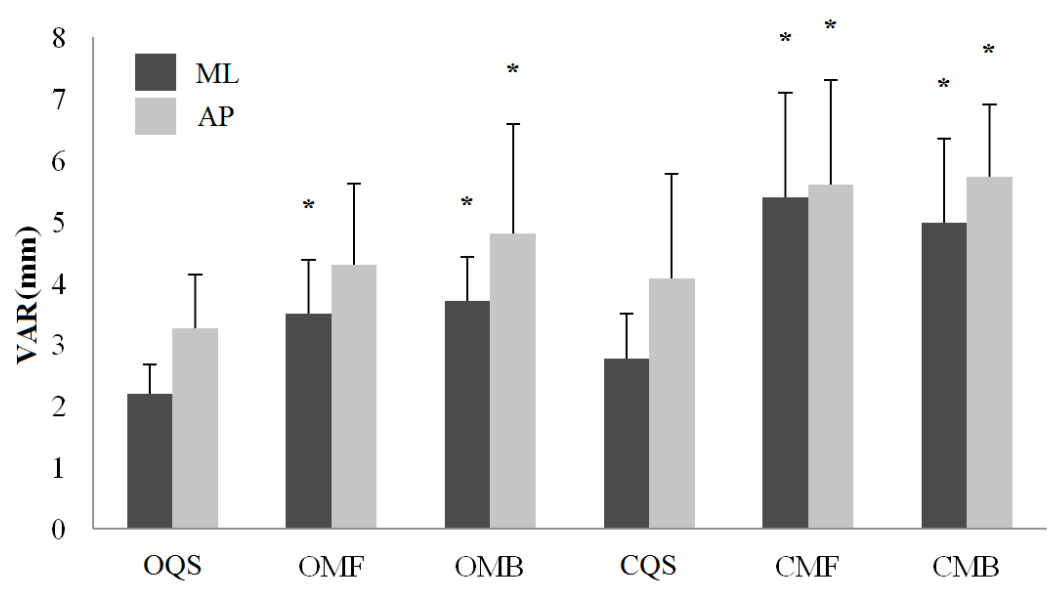

Figure 2. Mean values (SD) of COP variability (VAR). Vertical bars indicate standard deviation (OQS - eyes open quiet stance, OMF - eyes open maximum forward tilt, OMB - eyes open maximum backward tilt, CQS - eyes closed quiet stance,

CMF - eyes closed maximum forward tilt, CMB - eyes closed maximum backward tilt, ML - medial-lateral plane, AP - anterior-posterior plane. Asterisks indicate significant differences $(p<0.05)$ between conditions.

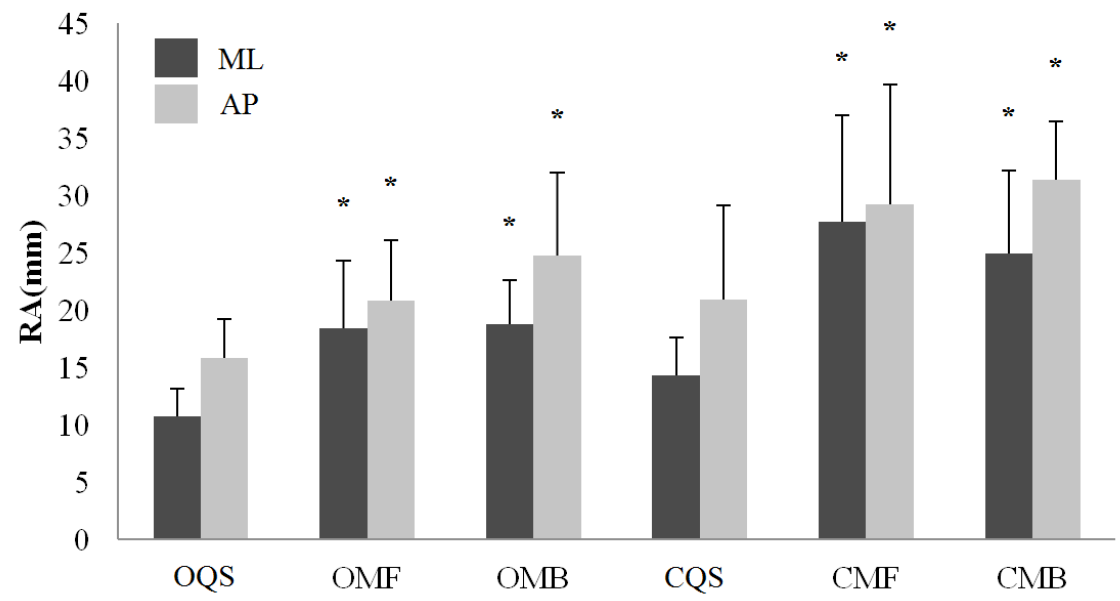

Figure 3. Mean values (SD) of COP range (RA). Vertical bars indicate standard deviation (OQS - eyes open quiet stance,

OMF - eyes open maximum forward tilt, OMB - eyes open maximum backward tilt, CQS - eyes closed quiet stance,

$\mathrm{CMF}$ - eyes closed maximum forward tilt, $\mathrm{CMB}$ - eyes closed maximum backward tilt, ML - medial-lateral plane,

AP - anterior-posterior plane. Asterisks indicate significant differences $(p<0.05)$ between conditions.

\section{COP Range}

There were three main effects: VISION $(F(1,15)=80.65$, $p<0.001)$, POSITION $(F(2,30)=23.32, p<0.001)$ and $\operatorname{PLANE}(F(1,15)=44.32, p<0.001)$. Range was higher with eyes closed, at the stability borders and in AP plane. There was also the interaction VISION x POSITION $(F(2,30)=3.53$, $p<0.05)$. The latter interaction reflected higher effect of standing at the stability borders with eyes closed. Post-hoc test showed that all changes in range were significant $(p<0.01)$. Mean values of COP range are shown in Figure 3 .

\section{COP Mean Velocity}

There were three main effects: VISION $(F(1,15)=71.25$, $p<0.001)$, POSITION $(\mathrm{F}(2,30)=48.15 p<0.001)$ and PLANE $(F(1,15)=195.67, p<0.001)$. Mean velocity was higher with eyes closed, at the stability borders and in the AP plane. In addition, there were three interactions: (1) POSITION $x \operatorname{PLANE}(F(2,30)=22.04 p<0.001)$ indicating higher effect of standing at the stability borders in the AP plane; (2) VISION $x$ PLANE $(F(1,15)=5.75, p<0.03)$, reflecting higher effect of eyes closure in the AP plane; (3) VISION x POSITION $(F(2,30)=8.29, p<0.002)$ showing higher effect of standing at the stability borders with eyes closed. Tukey test showed that the all changes in mean velocity were significant $(p<0.01)$. Mean values of COP velocity are shown in Figure 4.

\section{COP Frequency}

There were two main effects on the COP frequency: $\operatorname{VISION}(F(1,15)=5.27, p<0.04)$ and PLANE $(F(1,15)=35.74$, $p<0.001)$. Frequency was larger with eyes open and in the AP plane plane. There was also a POSITION x PLANE interaction $(\mathrm{F}(2,30)=7.20, p<0.003)$ indicating that the tilted position increased sway frequency in the AP plane and decreased in the ML plane. Tukey test showed that the change in frequency was significant only during the forward tilt in the frontal plane with eyes closed $(p<0.02)$. Mean values of COP frequency are shown in Figure 5.

\section{COP Sample Entropy}

There were three main effects: VISION $(F(1,15)=24.82$, $p<0.001)$, POSITION $(F(2,30)=6.53, p<0.005)$ and PLANE $(F(1,15)=46.97, p<0.001)$. Entropy values were smaller with eyes closed, at the stability borders and in the ML plane. There was also one interaction POSITION x PLANE $(F(2,30)$ 


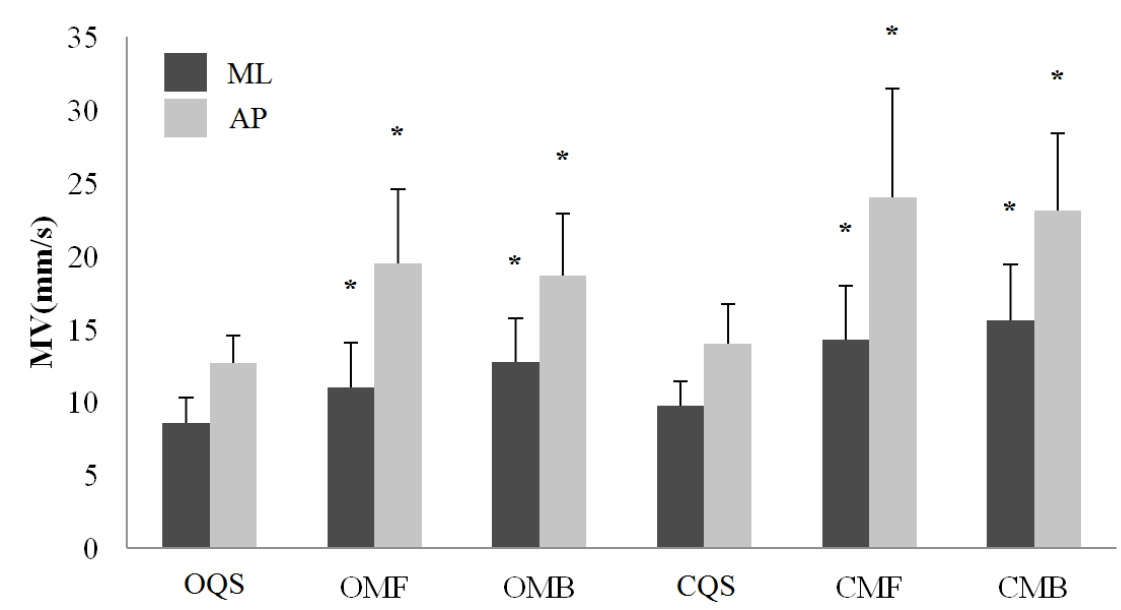

Figure 4. Mean values (SD) of COP mean velocity (MV). Vertical bars indicate standard deviation (OQS - eyes open quiet stance, OMF - eyes open maximum forward tilt, OMB - eyes open maximum backward tilt, CQS - eyes closed quiet stance, CMF - eyes closed maximum forward tilt, CMB - eyes closed maximum backward tilt, ML - medial-lateral plane, AP - anterior-posterior plane. Asterisks indicate significant differences $(p<0.05)$ between conditions

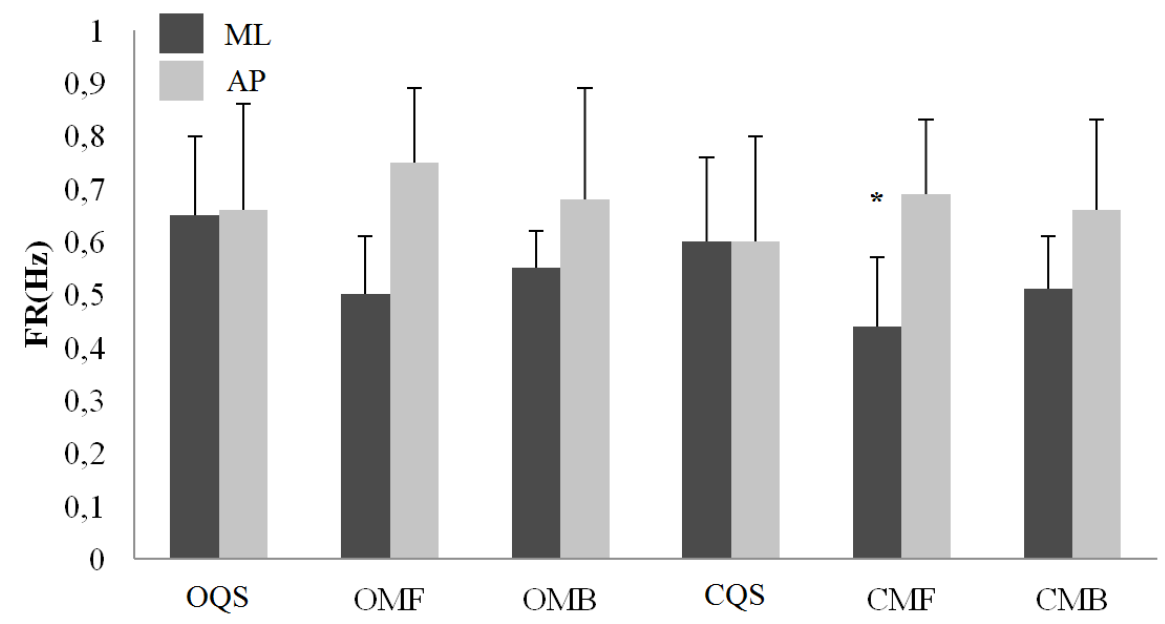

Figure 5. Mean values (SD) of COP frequency (FR). Vertical bars indicate standard deviation (OQS - eyes open quiet stance, OMF - eyes open maximum forward tilt, OMB - eyes open maximum backward tilt, CQS - eyes closed quiet stance,

$\mathrm{CMF}$ - eyes closed maximum forward tilt, $\mathrm{CMB}$ - eyes closed maximum backward tilt, $\mathrm{ML}$ - medial-lateral plane, AP - anterior-posterior plane. Asterisks indicate significant differences $(p<0.05)$ between conditions

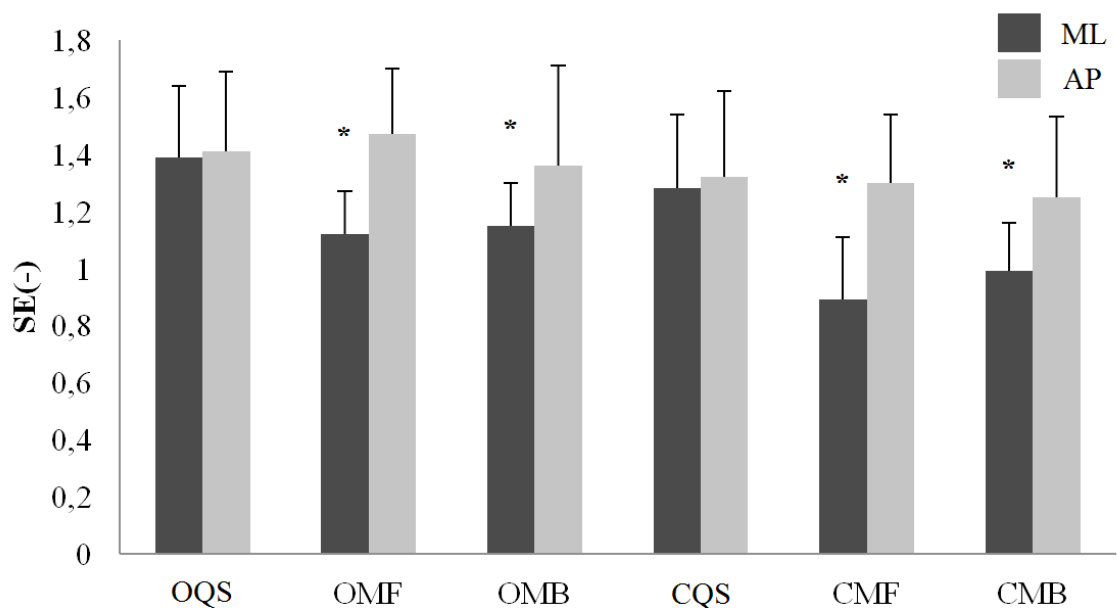

Figure 6. Mean values (SD) of COP sample entropy (SE). Vertical bars indicate standard deviation (OQS - eyes open quiet stance, OMF - eyes open maximum forward tilt, OMB - eyes open maximum backward tilt, CQS - eyes closed quiet stance,

CMF - eyes closed maximum forward tilt, CMB - eyes closed maximum backward tilt, ML - medial-lateral plane, AP - anterior-posterior plane. Asterisks indicate significant differences $(p<0.05)$ between conditions 


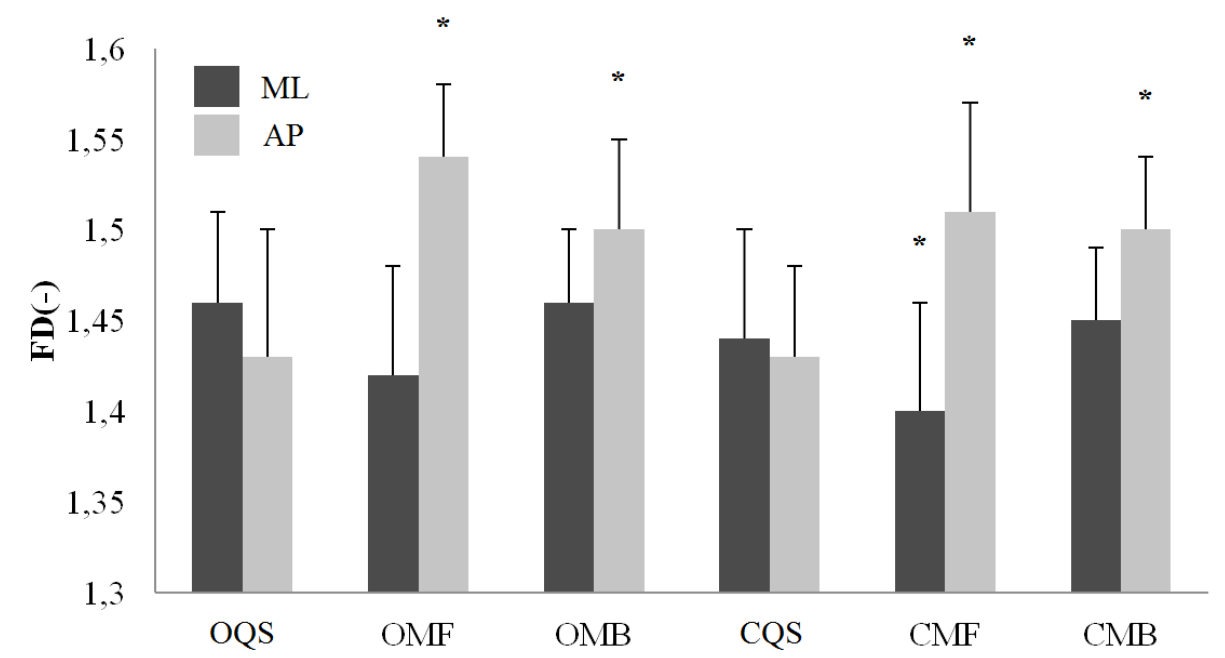

Figure 7. Mean values (SD) of COP fractal dimension (FD). Vertical bars indicate standard deviation (OQS - eyes open quiet stance, OMF - eyes open maximum forward tilt, OMB - eyes open maximum backward tilt, CQS - eyes closed quiet stance,

$\mathrm{CMF}$ - eyes closed maximum forward tilt, $\mathrm{CMB}$ - eyes closed maximum backward tilt, ML - medial-lateral plane, AP - anterior-posterior plane. Asterisks indicate significant differences $(p<0.05)$ between conditions

$=6.01 p<0.007)$ exibiting that COP sample entropy due to the body tilt decrased in the frontal plane only. Tukey test showed that the changes in entropy were significant only during the body tilt in the ML plane with eyes closed $(p<0.01)$. Mean values of COP sample entropy are shown in Figure 6. COP Fractal Dimension

There were main effects of PLANE $(F(1,15)=34.61, p<$ $0.001)$ and POSITION $(\mathrm{F}(2,30)=10.65, p<0.001)$. Fractal dimension weas higher in the AP plane and in the tilted positions. The POSITION x PLANE interaction $(F(2,30)=24.98$, $p<0.001$ ) accounted for different changes in fractal dimension in both planes due to body tilt. Tukey test disclosed the details: (1) both tilting directions increased fractality in the same way in the AP plane; (2) in the ML plane the decrease in fractality was caused only by the forward tilt. Mean values of COP fractal dimension are shown in Figure 7.

\section{Discussion}

The purpose of this study was to evaluate the effects of proximity to stability borders and availability of vision on postural performance and strategies in young soccer players. The proximity to the stability borders was accomplished by maximal leaning of the erect body in the fore (forward tilt) and aft (backward tilt) directions preserving the full contact of the feet with the support surface. Similar investigations regarding the limits of stability were performed by numerous authors $[10,16,17]$, however their purpose was always to assess the maximal excursions toward the stability borders or some other measures of this task like directional control, endpoint excursions and movement (to the target) velocity [18].

The present results are an important extension of previous studies that addresses changes in postural performance and strategies which were brought about by standing close to the stability borders. Three findings are of particular interest. First, consistent with expectations, postural performance measured by COP variability and velocity was similarly deteriorated by forward and backward tilt and the decline in performance was greater without vision. Second, sway irregularity decreased in the ML plane in both visual conditions and for both tilts yet, it did not show any changes in the AP plane. And third, sway fractality increased in the AP plane in both visual conditions and for both tilts, while it decreased in the ML plane for the forward tilt only regardless the visual conditions.

Deteriorated postural performance at the stability borders was expected because a significantly decreased stability margin during body tilting must have obstructed the exploratory and performatory function of the spontaneous sway mainly due to very limited time to counteract any postural perturbations. The following discussion aspires to elucidate which postural strategies were used and why they were selected to cope with the situational challenge.

As expected, postural strategies used by our subjects while maintaining maximal voluntary body tilts were different from those exhibited in quiet stance. The associated changes in the COP entropy revealed significant decrease in sway irregularity in the ML plane without any variations in the AP plane. Entropy of sway has been most commonly proposed to reflect the interplay between unconscious and voluntary postural control with the former linked to the more irregular time-series that account for a lesser attentional involvement and more automaticity, while the latter being characteristic of a less automatic regulation with a larger attention investment $[19,20]$. The results of this study seem to be well accounted for by this description of entropy, since performing maximal forward and backward body tilt certainly requires deliberate involvement of attention as compared to highly spontaneous quiet standing. However, in view of a large decrease in the ML entropy and no changes in the AP entropy, a question arises as to where or why the large portion of attention was invested?

If it was only for an alert control of the tilting task (in terms of fall avoidance and preserving the constant contact of the feet with supporting surface) we should have observed a drop in the AP entropy rather as it was the AP plane where the danger of losing balance and the feet contact conditions applied. Contrary to this expectation, complying with the task conditions was not an attention demanding task, at least in the group of young soccer players, what is confirmed by the lack of changes in the AP entropy. In an attempt to disentangle the mystery of the high demand for attention in the ML plane, it is worth noting the results of Błaszczyk et al [21] who compared limb load asymmetry in young and elderly subjects in quiet stance with normal and occluded vision. The asymmetry was larger in the elderly and this difference increased 
after eyes closure. Błaszczyk et al [21] interpreted this finding as preparatory limb unload strategy which could significantly shorten reaction time in balance recovery. In young people standing quietly this strategy is unnecessary due to large distance to the stability borders. However, while tilting at the borders, where almost no margin of stability is left and time to contact with the border is close to zero, a similar compensatory behavior seems plausible. Having used only one force platform we do not know if such an unloading asymmetry was actually completed. Still, we can assume that the subjects were aware of this problem and focused attention on precursory selection of the leading and back leg. To further elucidate this peculiarity an impending experiment with deliberate limb asymmetry is warranted.

Sway fractal dimension behaved in a different way than sway entropy during maximal body tilts. Fractal dimension significantly increased in the AP plane during the fore and aft tilt. On the other hand, in the ML plane it decreased to a lesser extent during the fore tilt but did not change during the aft tilt. The interpretation of the FD relies on the interplay between stability and adaptability (flexibility), with lower values characteristic for better stability and higher for better adaptability $[22,23]$. It is also thought that the increase or decrease in the FD values is associated with releasing or freezing of degrees of freedom (DoF), respectively. Of course, there is a considerable relationship between these both notions, with stability related to limited DoF, and flexibility to abundant DoF.

Based on these interpretations, the large increase in the FD of sway in the AP plane accounted for deteriorated stability due to a negligible stability margin that offered very poor and insufficient protection against any unexpected perturbation. On the other hand, the need to perform tilting task in a satisfactory manner might have influenced the mode of postural control by more frequently using hip strategy. The effect of the latter strategy can be identified in significantly increased COM MV in the AP plane. This makes an additional degree of freedom in comparison with quiet stance which is typically performed without involving movements at the hips. In sum, we may describe postural control during tilting in the AP plane as not only less stable but also possessing better flexibility.

In contrast, forward tilt in the ML plane decreased sway fractality which accounts for improved stability. We believe that this improvement may be due to the same process of prearranged selection of leading and back leg that was mentioned while discussing changes in sway entropy. Having selected the role of both legs and the respective weight distribution in case the stepping strategy is required, the participants may use this distribution as an extra set point for postural control. The additional information of this kind, though more or less imaginary, may well support the overall postural performance restricting the body excursion to the neighborhood of the reference point and thus stabilize the body. Why a similar occurrence was absent during the backward tilt, may be only subject to speculation. It is common knowledge that leaning backwards is practically not trained and much less frequently used than leaning forward. Thus, even physically fit subjects performing this rare task may be unable to benefit from the ancillary input described above.

As regards the effect of visual input on postural control, our results showed that, although the overall pattern of changes was similar with eyes open and closed, the lack of vision deteriorated postural control. This observation is at variance with the results of Bieć and Kuczyński [6] who reported no effect of visual input on bipedal stance in a very similar group of 13-year old soccer players. To clarify this issue, we thor- oughly compared both groups and disclosed significant differences between them. The soccer players in [6] were a rigorously selected group of candidates at the age of 7 years who have been training at the Polish premier league facilities and their postural control was investigated at the end of training season. On the other hand, the soccer players in the present study were mainly volunteers from the neighborhood of a third league facilities whose postural control was investigated just before the training season after summer holidays. These important differences may help elucidate factors which affect the quality of low reliance of balance on vision that is often associated with soccer players.

\section{Limitations}

This study should be viewed in light of some limitations. The number of participants was relatively small and they were selected from one soccer club only which makes the generalization of results restricted. There was also no comparison group.

\section{Conclusion}

Forward and backward tilt deteriorated postural performance in a similar way. However their effects on postural strategies were direction dependent. The strategies in the frontal plane indicated increased investment of attention, lower automaticity and more stable control. In contrast, the sagittal strategies displayed decreased stability with no changes in automaticity. Such inter-plane differences suggest specific allocation of the critical postural tasks in response to the threat of fall. It is plausible that, due to complexity of these reciprocal relationships, testing postural control near to stability limits may better differentiate specific groups and experimental manipulations than testing the limits of stability only. The low reliance of postural control on vision, often attributed to soccer players may be not a permanent attribute but may depend on the actual involvement in training and may develop in relation to individual abilities.

\section{Disclosure statement}

No author has any financial interest or received any financial benefit from this research.

\section{Conflict of interest}

The authors state no conflict of interest.

\section{References}

1. Bressel E, Yonker JC, Kras J, Heath EM. Comparison of static and dynamic balance in female collegiate soccer, basketball, and gymnastics athletes. J Athl Train. 2007; 42(1):42-46.

2. Paillard T. Plasticity of the postural function to sport and/ or motor experience. Neurosci Biobehav Rev. 2017;72: 129-152; doi: 10.1016/j.neubiorev.2016.11.015.

3. Ben Moussa AZ, Zouita S, Dziri C, Ben Salah FZ. Postural control in Tunisian soccer players. Sci Sports. 2012;27(1): 54-56; doi: https://doi.org/10.1016/j.scispo.2011.03.006.

4. Ricotti L, Rigosa J, Niosi A, Menciassi A. Analysis of balance, rapidity, force and reaction times of soccer players at different levels of competition. Plos one. 2013;8(10): 1-21; doi: https://doi.org/10.1371/journal.pone.0077264.

5. McGuine TA, Keene JS. The effect of balance training program on the risk of ankle sprains in high school athletes. Am J Sports Med. 2006;34(7):1103-1111. 
6. Bieć E, Kuczyński M. Postural control in 13-year-old soccer players. Eur J Appl Physiol. 2010;110(4);703-708; doi: 10.1007/s00421-010-1551-2.

7. Ricotti L, Ravaschio A. Break dance significantly increases static balance in 9 years-old soccer players. Gait Posture. 2011;33(3):462-465; doi: https://doi.org/10.1016/j. gaitpost.2010.12.026.

8. Faraldo-García A, Santos-Pérez S, Crujeiras R, Soto-Varela $A$. Postural changes associated with ageing on the sensory organization test and the limits of stability in healthy subjects. Auris Nasus Larynx. 2016;43(2):149_ 154; doi:10.1016/j.anl.2015.07.001

9. Kasahara S, Saito H, Anjiki T, Osanai H. The effect of aging on vertical postural control during the forward and backward shift of the center of pressure. Gait Posture. 2015;42(4):448-454; doi:10.1016/j.gaitpost.2015.07.056

10. Juras G, Słomka K, Fredyk A, Sobota G, Bacik B. Evaluation of the Limits of Stability (LOS) Balance Test. J Hum Kinet. 2008;19:39-52.

11. Zamysłowska-Szmytke E, Śliwińska-Kowalska M. The examination of balance system in occupational medicine [in Polish]. Otorynolaryngologia. 2012;11(4):139-145.

12. Biegański P, Pyskir M, Pyskir J, Trela E, Hagner W. Postural stability of young soccer players compared to physically inactive peers [in Polish]. J Health Sci. 2013;3(13): 477-488.

13. Ricotti L. Static and dynamic balance in young athletes. J Hum Sport Exerc. 2011;6(4):616- 628.

14. Palmieri RM, Ingersoll CD, Stone MB, Krause BA. Center-of-pressure parameters used in the assessment of postural control. J Sport Rehabil. 2002;11:51-66.

15. Raymakers JA, Samson MM, Verhaar HJJ. The assessment of body sway and the choice of the stability parameter(s). Gait Posture. 2005;21:48-58.

16. Jonsson $E$, Henriksson M, Hirschfeld $H$. Does the functional reach test reflect stability limits in elderly people? J Rehabil Med. 2003;35(1):26-30.

17. Clark S, Iltis PW, Anthony CJ, Toews A. Comparison of older adult performance during the functional-reach and limits-of-stability tests. J Aging Phys Act. 2005:13(3): 266-75.

18. Pickerill ML, Harter RA. Validity and reliability of limits-ofstability testing: a comparison of 2 postural stability evaluation devices. J Athl Train. 2011;46(6):600-6.

19. Donker SF, Roerdink M, Greven AJ, Beek PJ. Regularity of center-of- pressure trajectories depends on the amount of attention invested in postural control. Exp Brain Res. 2007;181:1-11.

20. Borg FG, Laxåback G. Entropy of balance - some recent results. J Neuroeng Rehabil. 2010;7:38. doi: 10.1186/ 1743-0003-7-38

21. Blaszczyk JW, Prince F, Raiche M, Hébert R. Effect of ageing and vision on limb load asymmetry during quiet stance. J Biomech. 2000;33(10): 1243-8.

22. Błaszczyk JW, Klonowski W. Postural stability and fractal dynamics. Acta Neurobiol Exp (Wars). 2001;61(2):105-12.

23. Bieć E, Zima J, Wójtowicz D, Wojciechowska-Maszkowska B, Kręcisz K, Kuczyński M. Postural stability in young adults with Down syndrome in challenging conditions. Plos one. 2014;9(4):e94247:1-8; doi: https://doi.org/1 0.1371 /journal.pone.0094247. 